\title{
SOLUTIONS OF BOUNDED VARIATION OF THE FREDHOLM-STIELTJES INTEGRAL EQUATION*
}

\author{
BY L. J. PARADISO
}

The purpose of this note is to give conditions under which the Stieltjes integral equation

$$
\phi(x)=f(x)+\lambda \int_{a}^{b} K(x, y) d \phi(y)
$$

has a solution $\phi(x)$ of bounded variation.

In the first theorem conditions on $f(x)$ and $K(x, y)$ are given under which the method of successive substitutions yields a solution of bounded variation for a limited range of values of $\lambda$.

With further restrictions on $K(x, y)$, it is shown in the second theorem that the Fredholm method applies and the solution of bounded variation thus obtained is valid for all values of $\lambda$ except for the characteristic values.

Finally, an example is given to show that the more restrictive conditions on $K(x, y)$ given in the second theorem are not sufficient to make the problem a special case of that treated by Riesz. $†$

THEOREM 1. If

(a) $f(x)$ is of bounded variation, $a \leqq x \leqq b$,

(b) $K(x, y)$, defined and bounded on $R(a \leqq x \leqq b, a \leqq y \leqq b)$, is continuous in $y$ for each $x$ and has a total variation in $x$ for each $y, T_{K}(y)$, which is a bounded function of $y$ having the least upper bound $T_{K}$ and

$$
|\lambda|<1 / T_{K}
$$

then the function $\bar{\phi}(x)$ defined by the series

(2) $\bar{\phi}(x)=f(x)+\lambda \int_{a}^{b} K\left(x, y_{1}\right) d f\left(y_{1}\right)$

$$
+\lambda^{2} \int_{a}^{b} K\left(x, y_{1}\right) d \int_{a}^{b} K\left(y_{1}, y_{2}\right) d f\left(y_{2}\right)+\cdots
$$

is the unique solution of bounded variation of integral equation (1).

* Presented to the Society, December 31, 1930.

† F. Riesz, Über lineare Funktionalgleichungen, Acta Mathematica, vol. 41 (1918), pp. 71-98. 
This theorem is proved by the usual method of successive substitutions. Let the function $\psi(x)$ be defined by

$$
\psi(x)=\int_{a}^{b} K(x, y) d f(y) .
$$

Designate the total variations of $f(x)$ and $\psi(x)$ by $T_{f}$ and $T_{\psi}$ respectively and let $M \geqq|K(x, y)|$. Then we have

$$
|\psi(x)| \leqq M T_{f} \text { and } T_{\psi} \leqq T_{K} \cdot T_{f} .
$$

With the use of these inequalities it is found that series (2) obtained from equation (1) by repeated substitution converges absolutely and uniformly in $x$ for all values of $\lambda$ satisfying the inequality $|\lambda|<1 / T_{K}$. The function thus defined, $\bar{\phi}(x)$, is found by substitution to satisfy equation (1). That it is the unique solution follows as a consequence of the fact that the method of successive substitutions when applied to the homogeneous equation

$$
\phi(x)=\lambda \int_{a}^{b} K(x, y) d \phi(y)
$$

yields as its only solution $\phi(x) \equiv 0$.

THEOREM 2. If $f(x)$ is of bounded variation in the interval $(a, b)$ and $K(x, y)$ together with $\partial K(x, y) / \partial x$ are continuous functions of $x$ and $y$ in $R$, then there exists a unique solution $\phi(x)$ of bounded variation of equation (1) for all values of $\lambda$ except for the characteristic values.

This theorem can be proved by applying Fredholm's method to equation (1). However, we shall employ the following transformation* which reduces the problem to the solution of a Riemann integral equation. Let $\theta(x)=\phi(x)-f(x)$. Then equation (1) becomes

$$
\theta(x)=\lambda \int_{a}^{b} K(x, y) d f(y)+\lambda \int_{a}^{b} K(x, y) d \theta(y) .
$$

It is easily verified, with the given hypotheses on the functions involved, that each term of the right hand side of equation (3) is a continuous function of $x$ and possesses a continuous

* J. D. Tamarkin suggested to me the possibility of transforming equation (1) into a Riemann integral equation. 
derivative. Hence on placing $\lambda \int_{a}^{b} K(x, y) d f(y)=F(y)$, we obtain from equation (3) by differentiation

$$
\theta^{\prime}(x)=F^{\prime}(x)+\lambda \int_{a}^{b} \frac{\partial}{\partial x} K(x, y) \theta^{\prime}(y) d y .
$$

The function $F^{\prime}(x)$ is continuous, and, moreover, by hypothesis $\partial K(x, y) / \partial x$ is continuous in $x$ and $y$. Hence the Riemann integral equation (4) has a unique continuous solution $\theta^{\prime}(x)$ for all values of $\lambda$ except for the characteristic values. The unique solution of bounded variation $\phi(x)$ of equation (1) can thus be found.

An example. Let $\|f\|$ denote the maximum of the absolute value of the continuous function $f(x)$ in $(a, b)$. One of the conditions on the transformation

$$
T[f]=\int_{a}^{b} K(x, y) d f(y)
$$

as given by F. Riesz* is that there exists a constant $M$ such that for all continuous functions $f(x)$

$$
\|T[f]\| \leqq M\|f\| \text {. }
$$

The function $K(x, y)$ defined in the following example satisfies the conditions of Theorem 2 whereas inequality (5) given by Riesz is not satisfied by the transformation $T[f]$. We define $K(x, y)$ to be a function of one variable, thus:

$$
K(x, 0)=0, \quad K(x, y)=y \sin (\pi / y),(0<y \leqq 1) .
$$

We next define a sequence of continuous functions $f_{n}(y)$ bounded in $n$ and $y$. The $n$th member of this sequence is a function whose graph consists of a series of broken lines. These lines have a slope equal to zero in the interval $(0,1 /(n+1))$, while in the interval $(1 /(n+1), 1)$ they have a negative slope where the function $y \sin (\pi / y)$ is negative and a positive slope where this function is positive. Let $\delta_{i}$ denote 1 or 0 according as $i$ is odd or even. Then

$$
\begin{aligned}
f_{n}(y)=(-1)^{k} k(k+1) y+(-1)^{k+1} k+\delta_{k}, \\
1 /(k+1) \leqq y \leqq 1 / k,(k=\dot{1}, 2, \cdots, n),
\end{aligned}
$$

*F. Riesz, loc. cit., p. 72. 
and

$f_{n}(y)=\delta_{n}$,

$(0 \leqq y \leqq 1 /(n+1))$

From this definition we have

$$
\begin{aligned}
\frac{d f_{n}(y)}{d y}= & (-1)^{k} k(k+1), \\
& =0, \quad(1 /(k+1)<y<1 / k, k=1,2, \cdots, n),
\end{aligned}
$$

The transformation

$$
T\left[f_{n}\right]=\int_{0}^{1} y \sin (\pi / y) d f_{n}(y), \quad(n=1,2, \cdots),
$$

becomes from the definition of $f_{n}(y)$ and from (6)

$$
\begin{aligned}
T\left[f_{n}\right] & =\sum_{k=1}^{k=n} \int_{1 /(k+1)}^{1 / k} y \sin (\pi / y)(-1)^{k} k(k+1) d y \\
& =\sum_{k=1}^{k=n} k(k+1) \int_{1 /(k+1)}^{1 / k}(-1)^{k} y \sin (\pi / y) d y .
\end{aligned}
$$

The $\int_{1 / k+1)}^{1 / k} y \sin (\pi / y) d y$ is in absolute value greater than the area of the triangle whose vertices are at $(1 /(k+1), 0),(1 / k, 0)$, $\left[2 /(2 k+1),(-1)^{k} 2 /(2 k+1)\right]$. Since the area of this triangle is $1 /[k(k+1)(2 k+1)]$, we have

$$
\int_{1 /(k+1)}^{1 / k}(-1)^{k} y \sin (\pi / y) d y>1 /[k(k+1)(2 k+1)] .
$$

Consequently we get from (7)

$$
T\left[f_{n}\right]>\sum_{k=1}^{k=n} k(k+1) /[k(k+1)(2 k+1)]=\sum_{k=1}^{k=n} 1 /(2 k+1),
$$

from which it follows that $T\left[f_{n}\right]$ becomes infinite with $n$. Hence condition (5) is not satisfied and equation (1) is not a special case of that treated by F. Riesz.

Cornell University 